\title{
INVENTARIO DE LAS ASOCIACIONES ÁFIDO-PLANTA EN EL PERÚ
}

\section{CHECKLIST OF APHID-PLANT ASSOCIATIONS IN PERU}

\author{
Miguel Angel Delfino ${ }^{1}$
}

\section{Resumen}

La información sobre las asociaciones áfido-planta conocidas en el Perú y actualmente dispersas en la literatura entomológica se agrupó y ordenó sistemáticamente. Teniendo en cuenta la biología de los áfidos involucrados en estas asociaciones, algunas fueron consideradas erróneas y, por lo tanto, no consideradas. Los nombres científicos de los áfidos fueron actualizados. En total, se registran 460 asociaciones áfido-planta en las que están involucradas 66 especies de áfidos y 238 de plantas.

Palabras claves: inventario, áfidos, plantas, asociaciones

\begin{abstract}
Information on aphid-plant associations in Peru, available in many different papers was collected and systematized. Based on the biology of the aphids involved, some of the associations reported in the literature were considered erroneous and set aside. When necessary, the scientific names of the aphids were updated. Altogether, 460 aphid-plants associations involving 66 aphid species and 238 plant species are reported.
\end{abstract}

Key words: checklist, aphids, plants, associations

\section{Introducción}

Los áfidos o pulgones, debido a sus características biológicas e impacto económico en los cultivos, son considerados en todo el mundo como uno de los grupos entomológicos más importantes desde el punto de vista agronómico. Además de los daños directos que causan en los cultivos cuando aumentan sus poblaciones, también transmiten virus fitopatógenos que provocan disminución en los rendimientos $\mathrm{y}$ calidad de las semillas (Holman, 1980; Blackman \& Eastop, 2000).

Desde ya, ellos pasan de un cultivo a otro mediante vuelos migratorios y generalmente, las mismas especies, colonizan también la vegetación espontánea que forma parte de los distintos ambientes. Precisamente, estas plantas no cultivadas son fuente importante donde se originan los pulgones alados que colonizarán los respectivos cultivos y también suelen ser el reservorio de los virus que ellos mismos van a transmitir. Por otra parte, esta vegetación espontánea puede desempeñar una función benéfica, al constituir un importante reservorio de enemigos naturales de los pulgones, ya que las poblaciones de áfidos que ellas albergan favorecen una intensa multiplicación de parasitoides, predadores y agentes patógenos (Remaudiere \& Autrique, 1985).

$\mathrm{Si}$ bien los datos biológicos de las distintas especies de áfidos son muy útiles para su identificación, donde el más importante es la planta hospedante, también el conocimiento de aspectos ecológicos de los áfidos es necesario para el desarrollo de nuevas investigaciones que tendrán por objetivo la puesta a punto de sistemas de manejo económicamente razonables, aceptables para el ambiente y adaptadas a las condiciones del medio rural, lo que contribuirá eficázmente a la protección de los cultivos.

Por ello, el objetivo de este trabajo es agrupar sistemáticamente las asociaciones áfido-planta conocidas en el Perú y actualmente dispersas en la literatura entomológica, con el propósito de contribuir al conocimiento bioecológico de los áfidos. Aunque ardua y probablemente incompleta, ahora se estima necesaria su publicación, ya que otros investigadores podrán mejorarla y tal vez servirá de base para futuros estudios taxonómicos y ecológicos de la afidofauna del Perú.

\section{Materiales y Métodos}

Para conocer las asociaciones áfido-planta en el Perú, se tuvieron en cuenta los artículos publicados sobre áfidos encontrados en el Perú donde, además, se explicitan sus plantas hospedantes. Por ello, en los resultados no se dan a conocer todas las especies que componen la afidofauna peruana, ya que en algunos casos no se mencionó a la planta hospedante.

Los áfidos se presentan ordenados alfabéticamente por géneros $\mathrm{y}$, dentro de ellos, por especies según corresponda. Sus nombres científicos fueron actualizados, por lo que en algunas especies, antes de mencionar las plantas hospedantes, se hace referencia al nombre científico tal como fue citado para el Perú, especificando previamente el autor y año de dicha cita.

Las plantas hospedantes de cada especie también se mencionan en orden alfabético por géneros $y$ especies, seguidos de la correspondiente cita 
bibliográfica (autor y año) que hace referencia a dicha asociación. En algunos casos, se mencionó sólo el nombre vulgar de la planta hospedante, por lo que entonces se recogen estos nombres entre comillas, también ordenados alfabéticamente, luego de la lista de nombres científicos aludida. En algunos casos, se encontraron menciones de asociaciones erróneas, teniendo en cuenta la biología de la especie de áfido involucrada, la que entonces no fue incluída en este trabajo por considerar que se trataba de pulgones errantes que circunstancialmente se encontraron sobre la planta mencionada o bien podría tratarse de identificaciones incorrectas de los áfidos.

Para evitar la repetición de la misma asociación áfido-planta, aunque proveniente de otra área geográfica del Perú, generalmente se hace mención a la cita bibliográfica más antigua. Todo ello, debido a cuestiones de espacio y que además no es intención efectuar un tratamiento cuantitativo de las mismas, sobre todo teniendo en cuenta que abundan en la literatura entomológica del Perú referencias de estas asociaciones donde están involucradas con mucha frecuencia plantas introducidas como cultivos agrícolas u ornamentales.

\section{Resultados}

Se registran 460 asociaciones áfido-planta en las que están involucradas 66 especies de áfidos y 238 de plantas.

Acyrthosiphon bidenticola Smith, 1960 Bidens pilosa: Rubín de Celis \& Ortíz (1994).

Acyrthosiphon kondoi Shinji, 1938

"Leguminosas": Ortiz \& Raven (1991).

Acyrthosiphon malvae (Mosley, 1841)

Essig (1953): Aulacorthum pelargonii

Geranium domesticus: Guerra Turin (1975). Geranium sp.: Essig (1953). Solanum sp.: Essig (1953)

Acyrthosiphon pisum (Harris, 1776)

Medicago hispida: Valencia et al. (1975); Medicago sativa: Smith (1971); Trifolium pratensis: Valencia et al. (1975); "Frijol”: Smith (1971).

Amphorophora peruviana Essig, 1953

Coreopsis sp.: Smith (1971)

\section{Aphis craccivora Koch, 1854}

Essig (1953): Aphis medicaginis

Astragalus sp.: Essig (1953); Cassia aphylla: Essig (1953); Cassia sp.: Essig (1953); Gossypium barbadense: Celis \& Ortíz (1994); Lens sculenta: Celis \& Ortíz (1994); Medicago sativa: Celis \& Ortíz (1994); Phaseolus lunatus: Celis \& Ortíz (1994); Prosopis spp.: Núñez-Sacarías (1993); Prunus avium: Valencia \& Cárdenas (1973); Vicia faba: Essig (1953); “ajonjolí”: Ortíz (1980); “caupí”: Ortíz
(1980); "Labiatae": Da Silva et al. (1980); "Thorny legume": Essig (1953)

\section{Aphis fabae Scopoli, 1763}

Wille (1952): Aphis papaveris

Asparragus officinalis: Celis \& Ortíz (1994); Chenopodium murale: León Vicente (1992); Datura stromonium: León Vicente (1992); Lens sculentum: León Vicente (1992); Lycopersicum sculentum: León Vicente (1992); Phaseolus lunatus: Celis \& Ortíz (1994); Rumex crispus: León Vicente (1992); Solanum tuberosum: León Vicente (1992); Solanum nigrum: León Vicente (1992); Vigna unguiculata: Castillo Carrillo (1994); "Culantro": Wille (1952).

Aphis gossypii Glover, 1877

Annona sp.: Smith (1971); Baccharis glutinosa: Da Silva et al. (1980); Calycophyllum spruceanum: Couturier \& Gonzales Tangoa (1994); Casuarina sp.: Essig (1953); Citrullus vulgaris: Valencia \& Cárdenas (1973); Citrus sp.: Smith (1971); Cucurbita maxima: Essig (1953); Cucurbita pepo: Valencia \& Cárdenas (1973); Cucurbita sp.: Smith (1971); Cydonia oblonga: Valencia \& Cárdenas (1973); Cymbopogon citratus: Celis \& Ortíz (1994); Eupatorium sp.: Smith (1971); Gossypium barbadense: León Vicente (1992); Gossypium hirsutum: Da Silva et al. (1980); Gossypium sp.: Essig (1953); Hibiscus rosa-sinensis: Ortíz (1980); Hibiscus sp.: Smith (1971); Lens sculenta: Celis \& Ortíz (1994); Lycopersicum sculentum: León Vicente (1992); Medicago sativa: Celis \& Ortíz (1994); Mirabilis sp.: Valencia \& Cárdenas (1973); Nicotiana tabacum: Valencia \& Cárdenas (1973); Persea americana: Smith (1971); Phaseolus vulgaris: León Vicente (1992); Plantago major: Da Silva et al. (1980); Plumaria rubra: Smith (1971); Prunus avium: Valencia \& Cárdenas (1973); Punica granatum: Essig (1953); Pyrus malus: Da Silva et al. (1980); Rumex acetosella: Velarde (1973); Senecio vulgaris: Guerra Turin (1975); Solanum andigenum: Valencia \& Cárdenas (1973); Solanum muricatum: León Vicente (1992); Solanum topiro: Gil Bacilio \& Guevara Hilario (1994); Solanum tuberosum: Essig (1953); Sorghum halepense: Smith (1971); Theobroma cacao: Smith (1971); "Algodón": Smith (1971); "Algodonero": Wille (1952); "Araceae": Ortíz (1980); "Cítricos": Wille (1952); "Chilco": Smith (1971); "Cocona": Smith (1971); "Cucurbitáceas": Wille (1952); "Gerbera": Smith (1971); "Hierba Mora": Ortíz (1980); "Mango": Ortíz (1980); "Pallae": Smith (1971); "Pepinillo": Ortíz (1980); "Tabaco": Ortíz (1980); "Verdolaga": Ortíz (1980).

Aphis nasturtii Kaltenbach, 1843

Rumex crispus: Ortiz \& Raven (1991); Solanum tuberosum: Ortiz \& Raven (1991)

Aphis nerii Boyer de Fonscolombe, 1841 
Asclepias curassavica: Da Silva et al. (1980); Asclepias sp.: Essig (1953); Euphorbia sp.: Valencia \& Cárdenas (1973); Funastrum domboyanum: Da Silva et al. (1980); “Asclepiadaceae”: Ortíz (1980).

Aphis rumicis Linnaeus, 1758

Agave sp.: Essig (1953); Rumex sp.: Smith (1971); "Compositae": Essig (1953).

Aphis sarothamni Franssen, 1928

Cassia hokesiana: Valencia \& Cárdenas (1973).

Aphis spiraecola Patch, 1914

León Vicente (1992): Aphis citricola

Acalypha hispida: Smith (1971); Aphelandra sp.: Smith (1971); Bidens sp.: Smith (1971); Calycophyllum spruceanum: Couturier \& Gonzales Tangoa (1994); Citrus sinensis: Valencia \& Cárdenas (1973); Citrus sp.: Smith (1971); Cydonia oblonga: Valencia \& Cárdenas (1973); Cydonia sp.: Smith (1971); Erythrina sp.: Ortíz (1980); Euphorbia sp.: Ortíz (1980); Hibiscus sp.: Smith (1971); Lactuca sp.: Smith (1971); Magnolia sp.: Smith (1971); Malus bacata: Valencia \& Cárdenas (1973); Malus pumila: Smith (1971); Malus sp.: León Vicente (1992); Mangifera indica: Valencia \& Cárdenas (1973); Prunus domestica: Valencia \& Cárdenas (1973); Pyrus malus: Smith (1971); Salix sp.: Smith (1971); Spondias purpureas: Da Silva et al. (1980); "Chileo": Smith (1971); "Cocona": Smith (1971); "Compositae": Smith (1971); "Magnolia": Smith (1971); "Oleander": Smith (1971); "Pallae": Smith (1971); "Plum": Smith (1971); “Tabaco": Ortíz (1980).

Aulacorthum circumflexum (Buckton, 1876)

Brassica sinapsis alba: Valencia et al. (1975); Fragaria chiloensis: Valencia et al. (1975); Geranium domesticus: Valencia et al. (1975); Solanum excisishombeum?: Valencia et al. (1975).

Aulacorthum solani (Kaltenbach, 1843) Wille (1952): Macrosiphum solani

Eichornia sp.: Smith (1971); Geranium domesticus: Valencia et al. (1975); Geranium sp.: Smith (1971); Rosa sp.: Valencia et al. (1975); Rumex sp.: Smith (1971); "Aj'1": Wille (1952); “Compositae": Smith (1971).

Brachycaudus helichrysi (Kaltenbach, 1843) Anthemis spp.: Valencia et al. (1975); Baccharis lanceolata: Smith (1971); Calceolaria pinnata: Smith (1971); Calendula officinalis: Essig (1953); Calendula sp.: Smith (1971); Chrysanthemum maximum (?): Essig (1953); Chrysanthemum sp.: Essig (1953); Erigeron sp.: Smith (1971); Piqueria peruviana: Smith (1971); Senecio vulgaris: Valencia et al. (1975); Senecio sp.: Smith (1971); Solanum sp.: Smith (1971); "Borragas": Smith (1971); "Chamilco": Smith (1971); "Chetincoya": Smith (1971); "Coreopsis": Smith (1971); "Flor de Muerto": Smith (1971);
"Habas": Smith (1971); "Pacte (Legume?)": Smith (1971); "Plum": Smith (1971); "Suncho": Smith (1971).

Brevicoryne brassicae (Linnaeus, 1758)

Brassica campestris: Smith (1971); Brassica napus: Smith (1971); Brassica nigra: Essig (1953); Brassica oleraceae: Essig (1953); Brassica oleracea var. capitata: Valencia \& Cárdenas (1973); Brassica rapa: Essig (1953); Brassica sinapsis alba: Valencia et al. (1975); Brassica sp.: Essig (1953); Capparis spinosa: Essig (1953); Capsella bursa pastoris: Valencia et al. (1975); Diplotaxis murales: Valencia et al. (1975); Physallis peruviana: Smith (1971); Raphanus raphanistrum: Valencia et al. (1975); "Brassica": Smith (1971); "Cabbage": Smith (1971); "Coles": Wille (1952); "Coliflor": Smith (1971); "Mustard (Black?)": Smith (1971); "Nabo": Smith (1971); "Pallae": Smith (1971).

Capitophorus elaeagni (del Guercio, 1894)

Cynara scolymus: Valencia et al. (1975).

Carolinaia cyperi Ainslie, 1915

Cyperus esculentus: Valencia \& Cárdenas (1973); Cyperus sp.: Da Silva et al. (1980).

Cavariella aegopodii (Scopoli, 1763)

"Zanahoria": Valencia et al. (1975).

Cerataphis palmae (Ghesquière, 1934)

"Palma": Ortiz \& Raven (1991).

Chaetosiphon tetrarhodum (Walker, 1849)

Smith (1971): Pentatrichopus tetrarhodus

Rosa sp.: Smith (1971).

Chaitophorus leucomelas Koch, 1854 Populus sp.: Rubin de Celis (1992).

Dysaphis foeniculus (Theobald, 1923)

Daucus carota: Ortiz \& Raven (1991).

Eriosoma lanigerum (Hausmann, 1802) Pyrus malus: Essig (1953); "Apple": Smith (1971); "Manzano": Wille (1952); "Peral": Wille (1952).

Hyalopterus pruni (Geoffroy, 1762) Arundo donax: Smith (1971).

Hyperomyzus lactucae (Linnaeus, 1758) Carduus sp.: Smith (1971); Chicorium endivia: Valencia \& Cárdenas (1973); Lactuca sativa: Smith (1971); Sonchus asper: Valencia \& Cárdenas (1973); Sonchus oleraceus: Valencia et al. (1975); Sonchus sp.: Smith (1971); "Cannacho": Smith (1971).

Hysteroneura setariae (Thomas, 1878)

"Grass": Smith (1971).

Illinoia goldamaryae (Knowlton, 1938)

Da Silva et al. (1980): Masonaphis goldamaryae Smith (1971): Masonaphis goldamaryae

Dahlia variabilis: Da Silva et al. (1980); Dahlia sp.: Smith (1971); "Malva rosa": Smith (1971). 


\author{
Illinoia masoni (Knowlton, 1928) \\ Valencia \& Cárdenas (1973): Masonaphis masoni \\ Dahlia rosea: Valencia \& Cárdenas (1973).

\section{Lipaphis erysimi (Kaltenbach, 1843) \\ Essig (1953): Rhopalosiphum pseudobrassica Smith (1971): Lipaphis pseudobrassicae \\ Wille (1952): Rhopalosiphum pseudobrassicae} \\ Brassica campestris: Smith (1971); Brassica napus: \\ Valencia \& Cárdenas (1973); Brassica oleracea: Da \\ Silva et al. (1980); Mathiola hederacea: Essig (1953); \\ Mathiola incana: Smith (1971); Mathiola sp.: \\ Essig(1953); Physalis peruviana: Smith(1971); \\ "Coles": Wille (1952); "nabo": Ortíz (1980); \\ "Pallae": Smith (1971); "Rabanito": Ortíz(1980).
}

Macrosiphoniella sanborni (Gillette, 1908) Chrysanthemum sp.: Smith (1971).

Macrosiphum edrossi Essig, 1953

Urtica sp.: Essig (1953).

\section{Macrosiphum euphorbiae (Thomas, 1878) \\ Essig (1953): Macrosiphum solanifolii Wille (1952): Macrosiphum solanifolii}

Argemone mexicana: Valencia et al. (1975); Asclepias sp.: Essig (1953); Brassica campestris: Guerra Turin (1975); Brassica sinapsis alba: Valencia et al. (1975); Calendula officinalis: Essig (1953); Capsicum annuum: Smith (1971); Chenopodium quinoa: Valencia et al. (1975); Chenopodium sp.: Smith (1971); Chicorium endivia: Valencia \& Cárdenas (1973); Chrysanthemum frutescens: Essig (1953); Datura stramonium: Valencia \& Cárdenas (1973); Datura sp.: Essig (1953); Erodium cicutarium: Valencia et al. (1975); Glycine max: Smith (1971); Gossypium hirsutum: Da Silva et al. (1980); Gossypium sp.: Essig (1953); Ipomoea batata: Smith (1971); Ipomoea sp.: Smith (1971); Lactuca sativa: Smith (1971); Malva sp.: Essig (1953); Malvabiscus sp.: Smith (1971); Nasturtium sp.: Smith (1971); Nicandra physalodes: León Vicente (1992); Nicotiana sp.: Essig (1953); Papaver argemone: Valencia \& Cárdenas (1973); Phaseolus lunatus: Celis \& Ortíz (1994); Phaseolus vulgaris: Essig (1953); Punica granatum: Essig (1953); Pyrus malus: Valencia et al. (1975); Raphanus raphanistrum: Valencia et al. (1975); Rosa sp.: Smith (1971); Ruta graveolens: Smith (1971); Senecio vulgaris: Valencia et al. (1975); Solanum andigenum: Valencia \& Cárdenas (1973); Solanum excisishombeum: Valencia et al. (1975); Solanum tuberosum: Essig (1953); Solanum sp.: Essig (1953); “Ají": Wille (1952); "Beans": Smith (1971); "Camote": Sanchez (1988); "Carnation": Smith (1971); "Columbine": Smith (1971); "Compositae": Smith (1971); "Cotton": Smith (1971); "Frijol bayo": Smith (1971); "Manzano": Wille (1952); "Mora Dilla": Smith (1971); "Papa": Wille (1952); "Sweet Potatoes": Smith (1971); "Thorny shrub" (Leguminosae): Essig (1953).
Macrosiphum rosae (Linnaeus, 1758)

Rosa sp.: Smith (1971); Rosa spp.: Valencia et al. (1975); "Rosa": Wille (1952); "Rosas cultivadas": Essig (1953).

Melanaphis sacchari (Zehntner, 1897) Saccharum officinarum: Da Silva et al. (1980).

Metopolophium dirhodum (Walker, 1849) Andropogon saccharoides: Valencia et al. (1975); Avena fatua: Valencia et al. (1975); Avena sativa: Smith (1971); Hordeum vulgare: Valencia et al.(1975); Hordeum sp.: Smith (1971).

Myzaphis rosarum (Kaltenbach, 1843)

Rosa sp.: Smith (1971); Rosa spp.: Valencia et al. (1975).

Myzus ornatus Laing, 1932

Bidens pilosa: Valencia et al., (1975); Cicyos sp.: Guerra Turin (1975); Eupatorium glechnophyllum: Guerra Turin (1975); Euphorbia peplus: Smith (1971); Fuchsia sp.: Essig (1953); Hedera helix: Valencia et al. (1975); Medicago hispida: Valencia et al., (1975); Medicago lupulina: Valencia et al. (1975); Medicago sativa: Smith (1971); Mentha sp.: Smith (1971); Polygonum aviculare: Valencia et al. (1975); Rumex acetosella: Valencia et al. (1975); Rumex sp.: Smith (1971); Salvia sp.: Smith (1971); Solanum excisishombeum?: Valencia et al. (1975); Solanum sp.: Smith (1971); Stachys sp.: Valencia et al. (1975); Trifolium incarnatum: Smith (1971); Trifolium pratensis: Smith (1971); Verbena communis: Valencia et al. (1975); Vicia faba: Smith (1971); Viguiera pflanzii: Valencia et al. (1975); "Flor de Muerto": Smith (1971); "Palmareal": Smith (1971).

\section{Myzus persicae (Sulzer, 1776)}

Amaranthus sp.: Smith (1971); Anthirrinum majus: Valencia et al. (1975); Antirrhinum sp.: Smith (1971); Brassica napus: León Vicente (1992); Brassica oleracea: Da Silva et al. (1980); Brassica oleraceae capitata: Smith (1971); Brassica sinapsis alba: Valencia et al. (1975);Brassica sp.: Smith (1971); Calendula officinalis: Smith (1971); Capparis spinosa: Essig (1953); Capsicum annuum: León Vicente (1992); Capsicum frutescens: Da Silva et al. (1980); Carica papaya: Celis \& Ortíz (1994); Cataranthus rosea: Valencia \& Cárdenas (1973); Chenopodium quinoa: Valencia et al. (1975); Chenopodium sp.: Smith (1971); Cucurbita maxima: León Vicente (1992); Cucurbita pepo: Valencia et al. (1975); Cymbopogon citratus: Celis \& Ortíz (1994); Dahlia variabilis: Valencia et al. (1975); Datura sp.: Essig (1953); Datura stramonium: Smith (1971); Daucus carota: Smith (1971); Diplotaxis murale: Valencia et al. (1975); Dunnalia campanulata: Da Silva et al. (1980); Eupatorium sp.: Smith (1971); Euphorbia peplus: Smith (1971); Geranium sp.: Smith (1971); Hedera helix: Valencia et al. (1975); Hibiscus 
sp.: Smith (1971); Ipomoea aff. nations?: Valencia et al. (1975); Ipomoea batatas: León Vicente (1992); Ipomoea sp.: Smith (1971); Lens sculentum: León Vicente (1992); Lycopersicum sculentum: León Vicente (1992); Magnolia sp.: Smith (1971); Malva parviflora: Valencia et al. (1975); Malva sp.: Smith (1971); Nicotiana sp.: Essig (1953); Phlox sp.: Essig (1953); Physalis peruviana: Smith (1971); Piqueria peruviana: Smith (1971); Pisum sp.: Smith (1971); Raphanus raphanistrum: Valencia et al. (1975); Rosa sp.: León Vicente (1992); Rottsoelia exaltata: Ortíz (1980); Rumex acetosella: Valencia et al. (1975); Rumex sp.: Smith (1971); Sesamum orientale: Valencia \& Cárdenas (1973); Solanum melongena: Smith (1971); Solanum muricatum: Smith (1971); Solanum sp.: Essig

(1953); Solanum tuberosum: Essig (1953); Stachys sp.: Valencia et al. (1975); Triticum aestivum: Smith (1971); Vicia faba: Smith (1971); “Aj'1”: Wille (1952); "Ajonjoli": Smith (1971); "Apple": Smith (1971); "Beet": Smith (1971); "Chamilco": Smith (1971); "Coles": Wille (1952); "Coliflor": Smith (1971); "Flor de Muerto": Smith (1971); "Frijol": Smith (1971); "Hiccha": Smith (1971); "Malva Rosa": Smith (1971); "Mustard": Smith (1971); "Nabo": Ortíz (1980); "Papa": Wille (1952); "Quita-liccha": Smith (1971); "Thorny tree": Essig (1953); "Tomate": Wille (1952).

Nasonovia ribisnigri (Mosley, 1841)

Crepis capilaris: Valencia et al. (1975).

Neotoxoptera formosana (Takahashi, 1921) Allium cepa: Ortiz \& Raven (1991).

Pentalonia nigronervosa Coquerel, 1859 Musa sapientum: Smith (1971); "Banana": Smith (1971); "Plátanos": Ortíz (1980).

Phloeomyzus passerinii (Signoret, 1875) Populus sp.: Smith (1971).

Picturaphis brasiliensis (Moreira, 1925) Phaseolus vulgaris: Ortiz \& Raven (1991).

Rhodobium porosum (Sanderson, 1900) Rosa sp.: Smith (1971).

Rhopalosiphoninus staphyleae (Koch, 1854) Hedera helix: Valencia et al. (1975).

\section{Rhopalosiphum maidis (Fitch, 1856)} Wille (1952): Aphis maidis

Hordeum vulgare: Valencia \& Cárdenas (1973); Secale cereale: Da Silva et al. (1980); Sorghum halepense: Essig (1953); Sorghum vulgare: Valencia \& Cárdenas (1973); Zea mays: Essig (1953); “Caña de Azúcar": Wille (1952); "Cebada”: Ortíz, Rodriguez \& Sarmiento M.(1981); “Maíz": Wille (1952); “Trigo": Wille (1952).

Rhopalosiphum nymphaeae (Linnaeus, 1761) Canna sp.: Essig (1953).
Rhopalosiphum padi (Linnaeus, 1758) Wille (1952): Rhopalosiphum prunifoliae Essig (1953): Rhopalosiphum prunifoliae

Zea mays: Essig (1953); "Cebada": Ortíz, Rodriguez \& Sarmiento M.(1981); "Gras": Celis \& Ortíz (1994); "Trigo": Wille (1952).

Rhopalosiphum rufiabdominalis (Sasaki, 1899) Essig (1953): Rhopalosiphum splendens

Oryza sativa: Da Silva et al. (1980); "Tomate": Escajadillo \& Ortíz (1993).

Schizaphis graminum (Rondani, 1852) Wille (1952): Toxoptera graminum

"Cebada": Ortíz, Rodriguez \& Sarmiento M. (1981); “Trigo": Wille (1952); “Maíz": Wille (1952).

Sipha flava (Forbes, 1884)

Cymbopogum citratus: Da Silva et al. (1980); Sorghum halepense: Celis \& Ortíz (1994); "Caña de Azúcar": Wille (1952).

Sitobion avenae (Fabricius, 1775)

Valencia et al. (1975): Macrosiphum avenea Fabricius (Sic) Andropogon saccharoides: Valencia et al. (1975); Avena fatua: Valencia et al. (1975); Avena sativa: Valencia et al. (1975); Dactylis glomerata: Valencia et al. (1975); Hordeum vulgare: Valencia et al. (1975); "Avena": Valencia et al. (1975); "Cebada": Valencia et al. (1975); "Trigo": Valencia et al. (1975).

Sitobion salviae (Bartholomew, 1932)

Smith (1971): Macrosiphum (Sitobion) salviae

Salvia sp.: Smith (1971).

Tetraneura nigriabdominalis (Sasaki, 1899)

"Grass": Ortiz \& Raven (1991).

Therioaphis trifolii (Monell, 1882)

“Alfalfa": Ortíz \& Rubin de Celis (1992).

Toxoptera aurantii (Boyer de Fonscolombe, 1841) Citrus aurantifolia: Celis \& Ortíz (1994); Ruta graveolens: Celis \& Ortíz (1994); "Cafeto": Wille (1952); "Cítricos": Ortíz (1980); "Cítrus": Wille (1952); "Te": Wille (1952).

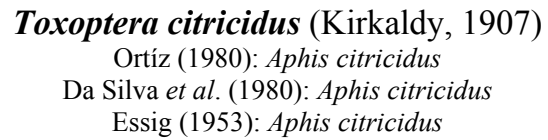

Citrus aurantifolia: Celis \& Ortíz (1994); Citrus grandis: Valencia \& Cárdenas (1973); Citrus limon: Smith (1971); Citrus nobilis: Valencia \& Cárdenas (1973); Citrus sinensis: Valencia \& Cárdenas (1973); Citrus sp.: Ortíz (1980); Citrus spp.: Essig (1953); "Bread plant": Smith (1971); "Cítricos": Beingolea (1967).

Tuberolachnus salignus (Gmelin, 1790)

Wille (1952): Pterochlorus viminalis Essig (1953): Tuberolachnus saligna

Cydonia oblonga: Valencia et al. (1975); Cydonia sp.: Smith (1971); Pyrus communis: Smith (1971); Salix humboldtiana: Wille (1952); Salix sp.: Essig (1953); "Manzano": Wille (1952); "Peral": Wille (1952). 
Uroleucon ambrosiae (Thomas, 1878)

Essig (1953): Macrosiphum ambrosiae

Chrysanthemum sp.: Essig (1953); Encelia sp.: Essig (1953); Gerbera jamesonii: Essig (1953); Nicotiana sp.: Essig (1953); Picris sp.?: Essig (1953).

Uroleucon erigeronensis (Thomas, 1878) Baccharis glutinosa: Da Silva et al. (1980); Sonchus oleraceus: Celis \& Ortíz (1994).

\section{Uroleucon sonchi (Linnaeus, 1767)} Smith (1971): Dactynotus sonchi

Ambrosia peruviana: Valencia \& Cárdenas (1973); Carduus sp.: Smith (1971); Lactuca sativa: Valencia \& Cárdenas (1973); Lactuca sp.: Smith (1971); Sonchus asper: Valencia \& Cárdenas (1973); Sonchus oleraceus: Da Silva et al. (1980); Spilanthes urens: Da Silva et al. (1980); Taraxacum officinale: Da Silva et al. (1980); "Turre macho": Da Silva et al. (1980).

\section{Uroleucon (Lambersius) sp.}

Capsicum pubescens: Valencia et al. (1975).

\section{Uroleucon sp.}

Nuda uliginosa: Valencia \& Cárdenas (1973).

Wahlgreniella nervata (Gillette, 1908)

"Rosa": Escajadillo \& Ortíz (1993).

\section{Agradecimientos}

A la Dra. Graciela Vilcapoma Segovia, Directora del Herbario Werbauer de la Universidad Nacional Agraria La Molina, Perú, por su paciencia en el suministro de la información botánica de las plantas involucradas en las asociaciones mencionadas con los áfidos.

\section{Literatura Citada}

Blackman R.L. \& Eastop V.F. 2000. Aphids on the World's Crops. An Identification and Information Guide. John Wiley \& Sons, Ltd. Chichester, England.

Castillo Carrillo P.S. 1994. Entomofauna asociada al cultivo de frijol caupí (Vigna unguiculata) en Tumbes. XXXVI Convención Nacional de Entomología, Libro de Resúmenes: 10. Iquitos, Perú.

Couturier G. \& Gonzales Tangoa J. 1994. Insectos plaga del árbol la Capirona cultivada en Iquitos. XXXVI Convención Nacional de Entomología, Libro de Resúmenes: 11. Iquitos, Perú.

Da Silva T.H., Ortíz M.S. \& Ojeda P.D. 1980. Aphididae (Homoptera) del Departamento de Lambayeque. Rev. per. Ent. 23 (1): 121-123.

Escarjadillo C. \& Ortíz Petrel M. 1993. El áfido Wahlgreniella nervata, nuevo registro para el Perú y tres áfidos adicionales para Ica. XXXV Convención Nacional de Entomología, Libro de Resúmenes: 3. Arequipa, Perú.

Essig E.O. 1953. Some new and noteworthy Aphidae from western and southern South America. Calif. Acad. Sci. Proc. 28 (3): 59-164.

Gil Bacilio J.L. \& Guevara Hilario E. 1994. Insectos plagas de la cocona (Solanum topiro) en Tingo María. XXXVI Convención Nacional de Entomología, Libro de Resúmenes: 12. Iquitos, Perú.

Guerra Turin C.D. 1975. Afidos (Homoptera - Aphididae), Hospederos y Enemigos Naturales en el Valle del Mantaro y Chocón. Tesis, Universidad Nacional del Centro del Perú, Huancayo - Perú.

Holman J. 1974. Los áfidos de Cuba. Instituto Cubano del Libro, La Habana, Cuba.

Núñez-Sacarías E. 1993. Insectos del algarrobo (Prosopis spp.) en el Perú: Costa Norte (Piura) y Costa Central (Ica). Rev. per. Ent. 36: 69-83.

Ortiz M.S. 1980. Aphididae (Homoptera) procedentes de ceja de selva: Tingo María (Huanuca-Perú). Rev. per. Ent. 23 (1): 119-120.

Ortiz M.S. \& Raven K.G. 1991. Ocho nuevos registros de Aphididae (Homoptera) para el Perú. Rev. per. Ent. 33: 123-124.

Ortiz M.S. Rodriguez A.C. \& Sarmiento M., J. 1981. Los áfidos (Homoptera: Aphididae) de la cebada (Hordeum vulgare $\mathrm{L}$.) en la sierra y costa centrales del Perú. Rev. per. Ent. 24 (1): 133-136.

Ortiz M.S. \& Rubín de Celis V.E. 1992. Therioaphis trifolii (Monell) (Homoptera: Aphididae: Drepanosiphinae), nuevo registro para el Perú. Rev. per. Ent. 35: 51-52.

Rubín de Celis V.E. 1992. Chaitophorus leucomelas Koch (Homoptera: Aphididae, Chaitophorinae), nuevo registro para Sudamérica. Rev. per. Ent. 35: 53.

Rubín de Celis \& Ortíz M. 1994. Aphididae (Insecta: Homoptera) procedentes de Canta (Lima - Perú). Biotempo. 1: 39-42.

Sánchez G.A. 1988. Empoasca spp. y Macrosiphum euphorbiae en camote de Rímac y Cañete, 1986. Rev. per. Ent. 31: 117-118.

Smith C.F. 1971. Survey of aphids on potatoes in Perú with notes on aphids on other plants. Second Report. North Carolina Agric. Mission. 31 pp. (Mimeografiado)

Valencia L., Guerra C. \& Gutarra F. 1975. Los áfidos (Homoptera: Aphididae) del Valle del Mantaro, plantas hospederas y enemigos naturales. Rev. per. Ent. 18(1): 90-97.

Valencia L. \& Cardenas D.C. 1973. Los áfidos (Homoptera: Aphididae) del Valle de Ica, sus plantas hospederas y enemigos naturales. Rev. per. Ent. 16 (1): 6-14.

Wille T.J.E. 1952. Entomología Agrícola del Perú. Ed. Junta de Sanidad Vegetal, Dirección General de Agricultura, Ministerio de Agricultura - Lima, Perú.

\footnotetext{
${ }^{1}$ Miguel Angel Delfino, Cátedra de Entomología, Facultad de C.E.F. y N., Universidad Nacional de Córdoba, Av. Vélez Sarsfield 299, 5000-Córdoba, Argentina, madelfino@arnet.com.ar
} 